\title{
The Silver Lining Methodology
}

\author{
José Miguel Castillo Chamorro ${ }^{1}, 2, \mathrm{PhD}$ \\ ${ }^{1}$ Universidad Internacional de la Rioja; \\ ${ }^{2}$ SOFTCAST Consulting S.L. Madrid. Spain
}

\begin{abstract}
The way in which Strategic planning is designed is different depending on the organization. For that reason, no standard procedures can be given to develop Strategic planning. However, the scenarios analysis method is used in any field or organization. We could define a scenario as a set of variables or events that describes a future situation. Additionally, the continuous irruption of new technologies invites us to carry out a revision of the old methodologies and procedures with the intention of starting an innovation process to make them more efficient. The challenge presented in this article consists of the use of the agents technology within a new methodological approach to envision future possible scenarios more quickly and more accurately than the classical methods we currently use.
\end{abstract}

Keywords - Foresight, Strategic Planning, Agents Technology, Methodology.

I. INTRODUCTION

Today the need for envisioning what is going to happen in the future is the same as centuries ago [1]. The developments in economy, industry, technology and society depend on the decisions we make in the present that, without any doubt, will have a direct influence in the future [2]. The more accurate the perception of what is going to happen in the future is the more correct these decisions will be.

Strategic planning is the subject responsible for the analysis of getting a future position within a future scenario. Consequently, the study and assessment of the future belong to the strategic planning analysis [3].

We should do this exercise of reflection very often with the aim of aligning our efforts towards the future final objectives whatever the application field is, entrepreneurial, industrial, social, technological, etc. The lack of this reflection exercise can unleash a chaos at any organization that will navigate with no direction as the scenario circumstances allow it to do it.

Social scenarios are very unsteady and are influenced by a big number of heterogeneous variables.

Changes in a situation will generate uncertainties; this implies the need of a new analysis when accomplishing the Strategic planning. Strategic planning does not eliminate uncertainty, but tries to reduce it with the aim of making decisions about the future with more possibilities of success.

The influence or modification of events in one scenario creates a variation in the global scenario with a major o minor intensity depending on the influence degree of each event within the scenario [4].

We can state that the success of the strategic planning depends on knowing with certain accuracy the events or variables that have a direct influence on the scenario and our capacity to eliminate, modify or foster these sensitive events. An approximate knowledge will always be better than a total ignorance or disregard for the strategic planning and its way to analyze the future.

Methodologies and techniques in Strategic planning are under the scope of IJIMAI since many of the new approaches are based on applied Artificial Intelligence. Namely, this article could be catalogued within one of the topics that are covered by IJIMAI: Heuristic and AI Planning Strategies and Tools.

This article is focused on the use of foresight to prevent and make an analysis of future scenarios by using the opinion of groups of experts.

The hypothesis that is introduced in this article consists of giving a new methodology to get future possible scenarios by using and treating adequately the opinion of human experts. Silver lining is the name given by the author of this article to the methodology. With this name the author tries to convey a positive view of hope to create and reach the future. The methodology not only includes the different phases and processes but also indicates the agent-based technologies that can be used to process human experts' opinions.

The article is divided into seven sections. A comparison between Forecast and Foresight are presented in section two. The different phases of the Silver lining methodology are introduced in section three. Section four illustrates a complete map of the processes of the Silver lining methodology. In section five the processes that can be developed by using the agent-based technologies are explained. An analysis of future works and applications is exposed in section six. Finally, section seven presents the conclusions of this article.

\section{Foresight vs. Forecast}

We can gather the different methods with scientific foundation to foresee the future in two groups: Technical and Anticipatory.

Within the Technical group, we can find mathematical models [5] that are developed to extrapolate past and present data in order to predict future results [6]. The study of tendencies permits us to approximate future situations in stable dynamic systems. Econometrics, demography and meteorology are sciences that can be included in this group. All these methods and scentific branches are included in the area of Forecast. That means that we can predict future scenario behaviour by means of algorithms or models able to predict future results or behaviours.

Technical prediction techniques are efficient in the creation of future scenarios based on stable dynamic systems in which tendencies of historical data are applied.

Unfortunately, social systems don't always behave in a stable way; normally they evolve in an unstable or chaotic way. Furthermore, when a situation includes a great deal of different and heterogeneous variables, technical prediction becomes complex and unaffordable [7].

The collapse of transportation, economic crisis, natural disasters, terrorist attacks, political scenarios, internal and external national 
conflicts are just a few of many examples of crisis scenarios which are difficult to estimate with techniques based upon technical prediction [8]. Normally, the crisis scenarios are created by an accumulation of events that would otherwise be ineffective in isolation; however when occurring together they create an unsustainable and critical scenario [9].

Anticipatory techniques try to avoid the problem of using technical prediction in unstable systems by using the opinion of a group of human experts [10]. The expert's opinion embodies relations among events or variables based on his/her personal experience; so complex relationships among heterogeneous events are mentally treated as a whole. The methods and techniques used to envision the future by using the opinion of groups of experts belong to the area of Foresight.

It is not until the mid of the twenty century when new techniques allow carrying out an anticipatory foresight, with the aim of giving a reliable and alternative solution to construct future possible scenarios in which the diversity, relationship and amount of variables make it impossible to process them by using forecast techniques.

Classical methodologies [1] [2] to carry out foresight exercises are still in use. It is worth mentioning the especial relevance of Godet's publications [11]. Nevertheless, it is important to remark that they are twenty years old and since that time AI technologies have evolved and reached a maturity level to be applicable in foresight studies and substitute statistical methods.

\section{Phases of Silver LiNING}

It is common that foresight exercises are linked. Departments or centers that carry out this kind of exercises make them in a continuous way. Normally there is a feedback between foresight exercises. The main objective of a foresight department is to generate information about the future in a continuous way, correcting or adjusting lack of accuracy. It is not a final objective for such departments to develop isolated foresight exercises.

The general phases of the Silver lining methodology are the following:

- Describing objectives. Initial Processes

- Consulting experts

- Processing experts' opinions

- Analysis of inferences

- Validating results.

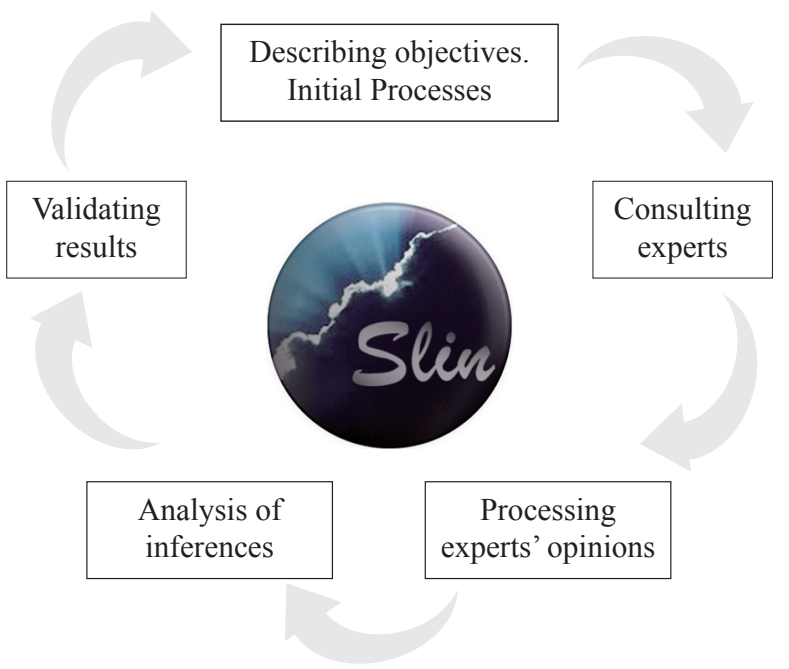

Fig. 1. Phases of the Silver lining methodology
In the first phase, 'Describing objectives' the aim of the foresight exercise is defined and the initial processes are prepared such as the selection of the temporal horizon, the events that may belong to the scenario and the selection of the group of experts. This phase can be recursive since many times it is necessary to re-define new objectives before starting the following phase.

Once the inquiries to the group of experts have been done, it is necessary to process all the information by using agent-based technologies. The result of this process has to be analyzed in order to study inferences or to give a new approach to the inquiries in case of any anomaly during the process.

The whole study finalizes with the validation phase that consists of checking the result with the scenario materialization through time. In the case that new events appear and they can influence the previewed scenario, it is necessary to accomplish a deep analysis of them to determine if a new foresight study is needed.

These five phases can be represented in an execution model that is illustrated in the following flowchart.

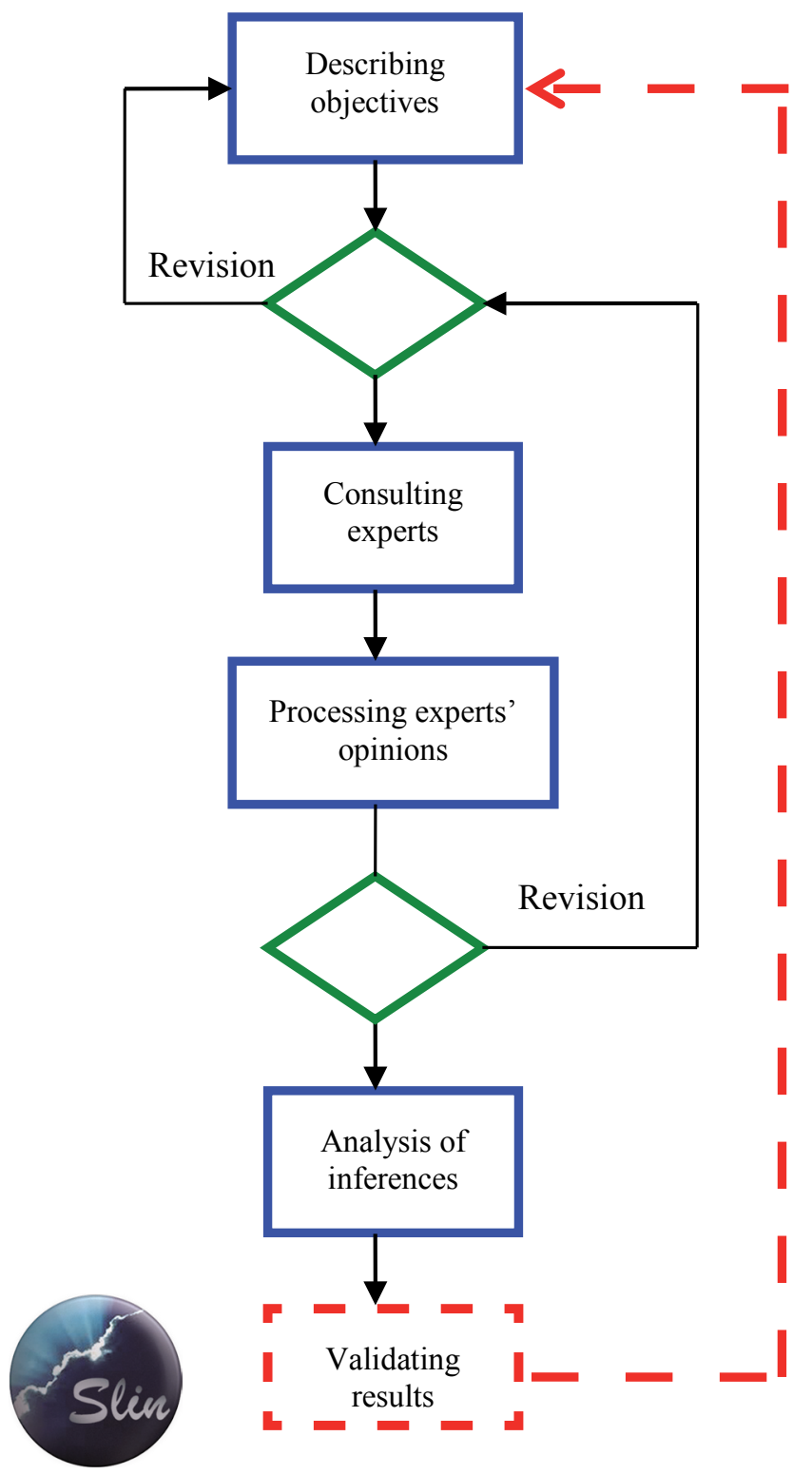

Fig. 2. Execution model flowchart 


\section{AgENT-BASED PROCESSES}

Strategic planning exercises are different depending on the application area, not only for the input data that is processed for planning but for the objectives to achieve as well as the internal rules used for the construction of different plans.

The technological evolution invites us to make a deep analysis of procedures used in foresight exercises with the aim of innovating in the case that the technology has reached the maturity level needed, facilitating more efficient results [12].

Regarding foresight studies in which human reasoning processes are dominant especially when experts gave their opinions about the possibility of achievement of a specific scenario or when gathering the opinion of all of them, the methods usually used are based on statistics and algebra. With the irruption of the Artificial Intelligence procedures we have the opportunity of developing an innovation exercise by substituting classical procedures for new technologies [13].

When doing a foresight exercise, the expression of the experts' opinions when qualifying a possible scenario and the global treatment of all answers reveal the necessity of using technologies that allow us to:

- Work with qualitative descriptions given individually by the experts, instead of quantitative answers expressed in terms of probabilities.

- Obtain the response of the group through the individual responses.

- Extrapolate the response of the whole group to envision the behavior of the rest of possible scenarios that are not possible to consult to the group of experts.

For the resolution of the problem of treating in a qualitative way the experts' opinions, it seems advisable the use of techniques based on Fuzzy Logic [14] that allow the elaboration of membership functions and rules to construct scenarios.

To get the answer of the group through the individual answers given by the experts, again it seems that Fuzzy Logic can provide a plausible solution.

Initially, to treat a set of heterogeneous variables with the aim of extrapolating the experts' opinions to the rest of scenarios within a specific domain, it seems that the use of Neural Networks [15] is adequate since it permits the classification of new scenarios after having been trained with the experts' opinions.

All these functionalities have to be developed under the Agency paradigm [16]. Agents are software entities able to develop certain cognitive capacity. That is to say that they simulate functionalities related to the human knowledge.

To construct agents in the planning environment (Strategic and Tactical), the MECIMPLAN methodology [17] could be adequate.

The following table matches the classic procedures to the substitutive technology.

TABLE 1. SUBSTITUTIVE TECHNOLOGIES

\begin{tabular}{|c|l|l|}
\hline Problem & Classic procedure & $\begin{array}{c}\text { Substitutive } \\
\text { technology }\end{array}$ \\
\hline $\begin{array}{c}\text { Getting individual } \\
\text { experts' opinions }\end{array}$ & $\begin{array}{l}\text {-Delphi (4 rounds) } \\
\text {-Answers in terms of } \\
\text { probability }\end{array}$ & $\begin{array}{l}\text {-Delphi (2 rounds) } \\
\text {-Answers in terms of } \\
\text { possibility }\end{array}$ \\
\hline $\begin{array}{c}\text { Getting opinions of the } \\
\text { group }\end{array}$ & $\begin{array}{l}\text {-Cross impacts } \\
\text { - Bayes theorem: } \\
\text { Conditional } \\
\text { probabilities }\end{array}$ & Fuzzy logic \\
\hline $\begin{array}{c}\text { Extrapolation of the rest } \\
\text { of possible scenarios } \\
\text { within the domain }\end{array}$ & Without solution & Neural Networks \\
\hline
\end{tabular}

\section{Map of Processes}

To develop each phase it is necessary to carry out a series of processes. The list of processes that belong to every phase is the following.

- Describing objectives. Initial Processes

Scope selection and temporal horizon

Stability study

Selection of participant

Selection experts group

Experts group evaluation

Selection of events

- Consulting experts

Questionnaires 1st round

Answers 1st round

Qualitative Process. 1st round

Sensitive events selection

Questionnaires 2nd round

Answers 2nd round

Qualitative Process. 2nd round

Sensitive events confirmation

- Processing experts' opinions

Rest scenarios Interpolation

Information analysis

- Analysis of inferences

Analysis of inferences

Final report draft

Proactive actions

- Validating results

Qualification metric

Validation of results

The majority of the Silver lining processes are developed in a sequential way.

The processes that belong to the first phase 'Describing objectives. Initial Processes' may be developed almost simultaneously since they are activities that do not require inputs from previous processes. Most of them begin once the study scope and temporal horizon are defined. The evaluation of the group of experts has to be done after the selection of the group components.

It is important to study the stability of the system. Dynamic systems evolve through time in three different ways: stable, unstable or chaotic. The more stable a system is the longer we can envision its behaviour. An unstable system evolves following certain tendencies between margins of stability, that is to say that the system behaviour is predictable within a margin. However, systems that evolve in a chaotic way are not predictable; in reality they are predictable but the result of the prediction has no value because many external variables could influence the system producing unexpected results.

To envision the future behaviour of a system by using the scenarios method we have to study if the system is evolving through time in a stable way or unstable with in a margin.

After finishing the first phase of Silver lining we should wonder if the exercise is well defined or if it is advisable to review some of the previous processes, such as reducing the scope of the study, increasing the number of experts or cancelling the study due to the instability of the system since the foresight study would yield uncertain results. 


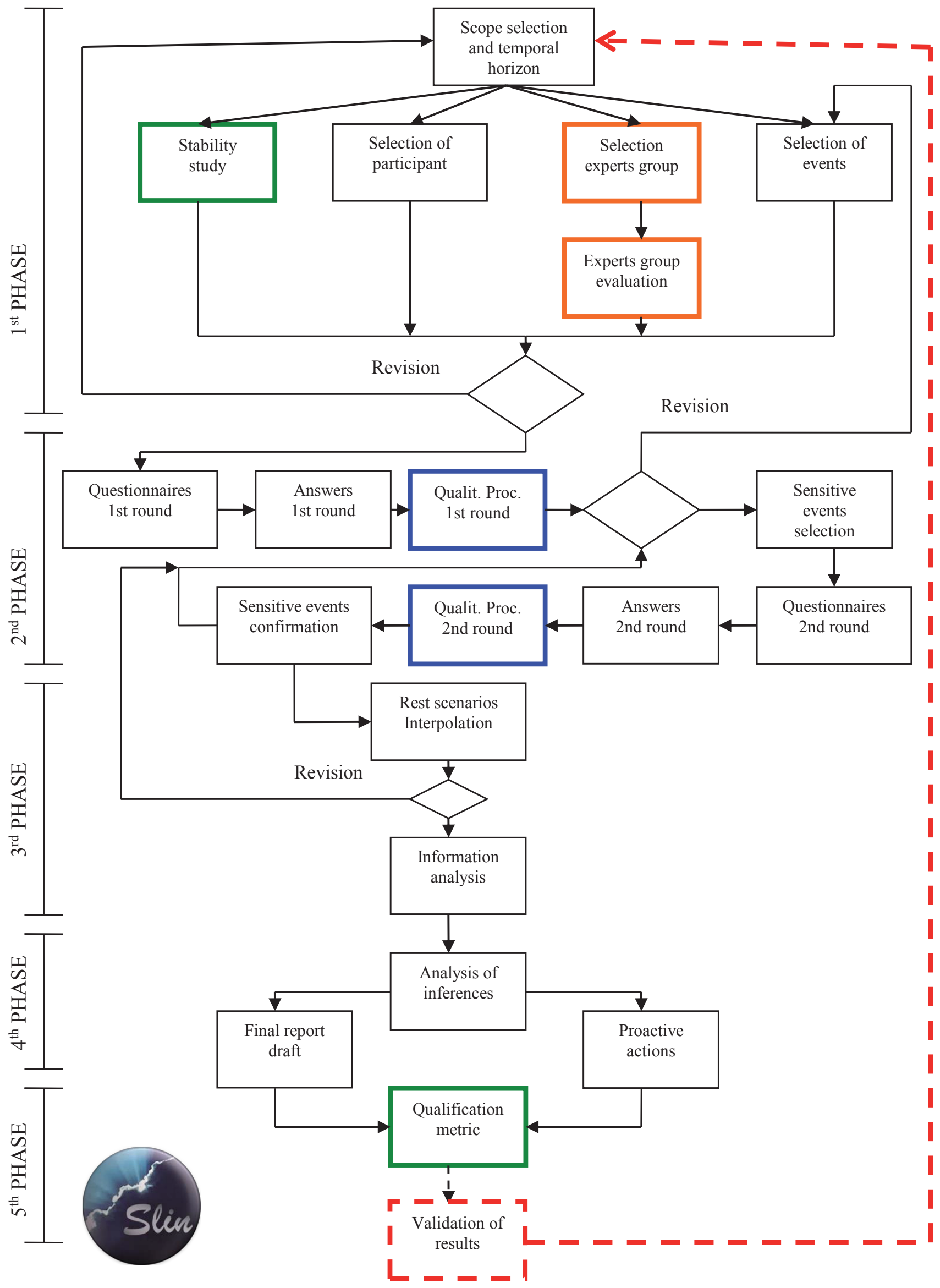

Fig. 3. Map of processes of the Silver lining methodology 
The processes that belong to the second phase of the methodology are sequential, that is to say that each one receives information from the previous one, with the exception of those cases in which an incorrect selection of the event has been detected or there is certain ambiguity in the interpretation of the inquiries by the experts. In that last case it will be necessary to define and select the events again in the first inquiry round as in the second. The Delphi technique [18] is used in this phase but with a variation regarding its classical use: we do not show the results of the first round to the group of experts contrasting opposite answers and trying to gather experts' opinions within the second and third quartile. We treat the expert' opinion as a rule within a fuzzy inference module. By processing this module we extract the opinion of the expert group as a whole and we get those events that are sensitive in the scenario. The second round of the Delphi technique is carried out in the case we need to confirm the events that have been identified as sensitive after the first round.

In both, the first and the second round we use Fuzzy Logic to process in a qualitative way the answers of the experts.

Within the third phase of the Silver lining the opinion of the experts is processed and the knowledge elicited from the experts is interpolated to the rest of scenarios not consulted.

The analysis of inferences is achieved in the fourth phase of the methodology. This phase is composed of three tasks that can be developed in parallel.

Finally, the fifth phase of Silver lining is focused on checking the quality and validity of the foresight exercise. The first process measures with a biased checklist the main activities done during the exercise, providing a tool to evaluate and compare the quality among foresight studies. The second process is extended through time with the aim of checking if the foreseen future is becoming a fact in the way it was expected. This last activity will boost new foresight studies in case the prediction was wrong or some new event has suddenly appeared changing the initial conditions of the original study.

\section{Applications and Future Works}

This article shows the main achievements after seven years of research trying to obtain a methodology that uses new technologies to allow us to carry out foresight studies in a more efficient way, rather than using classical methodologies or procedures.

Up to now the methodology has been applied successfully in more than five foresight exercises with Spanish think-tank centers [19]. Detailed and complete foresight studies in which the Silver lining methodology has been applied can be consulted in 'Instituto Español de Estudios Estratégicos' web page [20].

Foresight studies and the Silver lining methodology can be applied to simulate possible future scenarios at any field [21], such as evolution of technology, social behaviour, economy scenarios, crisis scenarios, decision in politics, etc.

Nevertheless, there is work left to be done in order to improve the results of foresight studies to envision future scenarios in the most realistic way.

Some of the future works that we are willing to face are listed below:

- Forecast and foresight working together: as it has been presented in section two of this article, forecast and foresight are seen currently as different methods to predict future scenarios; one based on mathematical models and the other processing complex relations with the most sophisticated computer 'the human brains of a group of experts'. It is necessary to study in depth the possibility of using with more flexibility both methods by means of integrating specific techniques in a single methodology that is able incorporate forecast and foresight, since in many occasions some variables that belong to a specific scenario can be affected through time by tendencies. The variable itself would have a double dependency, on one hand its tendency that could be calculated with a forecast study and on the other hand its complex relationship with the rest of variables, only predicted with a foresight study.

- Fostering software tools to support the use of foresight: Software tools permit the standardization and consolidation of procedures. The development of software tools that support foresight exercises with Silver lining would facilitate the methodology consolidation process.

- Validating experts' quality: The more right guesses of human experts the more realistic results in a foresight exercise. Therefore, the veracity of a foresight study is directly related to the quality of the each expert individually. It is vital that the expert be a real expert with a wide knowledge about the matter and with a high social intelligence level. It is necessary to study the kind of tests that an expert should pass in order to assure his quality as an expert.

- New solutions to the limitation of the number of events that belongs and describes a specific scenario: A number of seven events that could belong to a specific scenario would provide 128 possible scenarios. Silver lining permits the development of scenarios in cascade. Nevertheless, it would be advisable to study new ways of eliminating the limitations of the number of events.

In summary, the most important research lines that we are eager to face in a near future with interdisciplinary research groups are:

- Forecast and foresight working together

- Validating the expert's quality

- Reducing the limitation the number of events

\section{CONCLUSION}

Once we have reached the initial objectives that lead to the research project, as a conclusion of this article we can sum up the main achievements of this research project in three areas:

- From a methodological point of view

This research project provides the Silver lining methodology as a powerful tool that can guide the development of foresight studies. The methodology is agile, well-structured and applicable at any field that needs strategic planning.

\section{- From a technological point of view}

Nowadays, there are technologies that may substitute the mathematical-statistical methods that have been used up to now to develop the use of foresight. These technologies are mainly based on Fuzzy logic and Neural networks and are developed under the Agents paradigm. Processing natural language as the normal expression of human experts has a better understanding by the participants of a foresight study and they usually provide a more realistic solution.

- From the strategic planning point of view

Strategic planning is usually related to the long term, but there could be strategic decisions to make in the short term. For this reason, it is necessary to provide new solutions that working together with the latest technologies can give answers in the short term. This is the case of the result of this research.

\section{REFERENCES}

[1] Masini, E. (1994). Why Futures Studies? London: Ed. Grey Seal Books.

[2] Jarratt, J. (2003). From Scan to Plan: Integrating Trends into the StrategyMaking Process. American Society of Association Executives. 
[3] Bell, W. (1997). Foundations of Futures Studies. Human Science for a New Era: History, Purposes and Knowledge. Vol. 1. New Jersey: Transaction Publishers.

[4] Bas, E.; Guillo, M. (2013). Prospectiva e innovación (Vol. 1: visiones). Barcelona: Plaza y Valdés.

[5] Meadows, D. (1982). Groping in the dark; the first decade of Global Modelling. Bristol, John \& Sons.

[6] Castillo, J. M., et al. An Agent-Based Approach for Data Fusion in Homeland Security. International Journal of Interactive Multimedia and Artificial Intelligence, vol. 2, issue Special Issue on Improvements in Information Systems and Technologies , no. 3, pp. 44-49, 09/2013.

[7] Piedra-Calderón, J. C., et al. Global Collective Intelligence in Technological Societies: as a result of Collaborative Knowledge in Combination with Artificial Intelligence. International Journal of Interactive Multimedia and Artificial Intelligence, vol. 2, issue Regular Issue, no. 4, pp. 76-80, $12 / 2013$

[8] Castillo, J.M. (2010). A Technological Approach for Crisis Prevention. El Algarbe (Portugal): Proceedings of the e-Society International Congress.

[9] Castillo, J.M. (2011). An agent-based approach to envision the future. Lisbon (Portugal): Proceedings of the 8th International ISCRAM congress.

[10] Linstone, H.A. et al. (2010). Delphi: A brief look backward and forward. Technol. Forecast. Soc. Change, doi:10.1016/j.techfore.2010.09.011

[11] Godet, M. (1994). From anticipation to action. UNESCO Publishing.

[12] Castillo, J. M., et al. Prospecting the future with AI. International Journal of Interactive Multimedia and Artificial Intelligence, vol. 1, issue Special Issue on Business Intelligence and Semantic Web, no. 2, pp. 1-5, 12/2009.

[13] Castillo, J.M. (2012). Getting Experts'Agreement in Strategic Planning. Dubrovnik (Croatia): Proceedings of the Agreement Technologies International Congress.

[14] Zadeh, L. (1993). The role of fuzzy logic and soft computing in the conception and design of intelligent systems. Procedings of the 8th Austrian Artificial Intelligence Conference.

[15] Rosemblatt, F. (1958). The Perceptron: A probalistic model for information storage and organization in the brain. Psychological Review, 65, pags. 386-408.

[16] Castillo, J. M. A crystal ball made of agents. International Journal of Artificial Intelligence and Interactive Multimedia, vol. 1, issue A Direct Path to Intelligent Tools, no. 3, pp. 13-17, 12/2010.

[17] Castillo, J.M., et al. (2006). The MECIMPLAN Approach to Agent-based Strategic Planning. Hong Kong: Workshop Proceedings, IAT International Conference on Intelligent Agents Technology.

[18] Dalkey, N. C. (1972). The Delphi method: An experimental study of group opinion. Studies in the quality of life. Delphi and decision-making (pp. 13-54). Lexington Books.

[19] Castillo, J.M. (2012). Tecnología y Prospectiva: Un reto hecho realidad. Madrid: Rev. Instituto Español de Estudios Estratégicos. NIPO: 083-12238-7. Num 0 pags. $111-132$.

[20] http://www.ieee.es/investigacion/\#sub3

[21] Castillo, J.M. (2010). Simulating future crisis scenarios with MAS. Orlando (USA): Proceedings of the I/ITSEC International Congress.

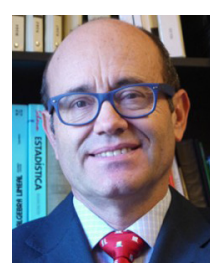

José Miguel Castillo Chamorro is the CEO of SoftCast Consulting. Currently he is Associate Professor at the Universidad Internacional de la Rioja in Madrid. From 2011 to 2013 he was the Director of the Area of Information Systems, training and decision making support in 'Tecnalia Research and Innovation'. From 2007 to 2011 he was Director of Division in European Virtual Engineering (Tech. Center). In 2001 he earned his $\mathrm{PhD}$ in Telecommunications from the Universidad Politécnica in Madrid. The same year he was awarded with the 'General Fernández Chicarro' prize by the Spanish Ministry of Defence for his work on Operations Research. He has a large experience leading projects in which simulation, artificial intelligence and project management are involved. From 1999 to 2013 he was Associate Professor at the Universidad Pontificia de Salamanca in Madrid. In 2007 he obtained his PhD in the Statistics and Operational Research department from the Universidad Rey Juan Carlos in Madrid. In June 2007 he was awarded with the prize on Research by the Spanish Ministry of Defence. In the same year he was awarded with the prize on Research on the field of Security by the Directorate of Civil Protection and Emergencies (Spanish Ministry of Interior). In July 2015 he earned his PhD in Sociology from the Universidad Pontificia de Salamanca. 